\title{
Systematic Performance Modeling and Characterization of Heterogeneous IP Networks*
}

\author{
Alessio Botta, Donato Emma, Antonio Pescapé, and Giorgio Ventre ${ }^{\dagger}$
}

\begin{abstract}
Accurate measurement and modeling of IP networks is essential for network design, planning, and management. Efforts are being made to detect the state of the network from end-to-end measurements using different techniques and paradigms. In this paper we present a novel concept to use in the modeling of real network scenarios under measurement and analysis. We called this new concept Service Condition. We explain our proposal's motivations and we show how to apply the Service Condition concept to the study of real heterogeneous network scenarios. To show the real applicability of our proposal results from a performance evaluation study over real heterogeneous networks (where the integration of LAN, UMTS and GPRS is present) are given.
\end{abstract}

\section{Introduction}

In last decade the networks have changed and the variety of applications and services that can be provided over packet switched networks has strongly increased and keeps on increasing. Therefore the need for accurate and innovative network monitoring and measurement paradigms has become an important challenge to be dealt with. The wide diffusion of smart devices with wireless connections (WLAN, GPRS, UMTS, ...) has triggered the development of new applications over new and complex network infrastructures. To study these novel network scenarios a precise framework and a systematic approach are needed.

There are several works on the performance evaluation of WLAN carried out by means of real measurements. A

* This work has been partially supported by the Italian Ministry for Education, University and Research (MIUR) in the framework of the WEB-MINDS FIRB Project, and PRIN 2004 QUASAR project, and finally by the E-NEXT IST European project. The authors would like to thank Salvatore Guadagno for his valuable support and hints.

$\dagger$ A. Botta is with the Consorzio Interuniversitario Nazionale per l'Informatica, Naples (Italy), abotta@napoli.consorzio-cini.it. D. Emma, A. Pescapé, and G. Ventre are with the Dipartimento di Informatica e Sistemistica, Università di Napoli "Federico II", Naples, Italy. \{doemma,pescape,ventre\}@unina.it. performance study on wireless LAN in a vehicular mobility scenario is presented in [1]. In [2] the performance of a real campus area network are measured moving on several parameters: received power, walls and floors separating two radio interfaces and finally interfering traffic. In [3] the authors present a comprehensive study on TCP and UDP behavior over WLAN taking into account radio hardware, device drivers and network protocols. [4] shows performance measurements carried out on a real MAN in order to evaluate the real throughput. In our opinion, all these cited works contain optimal research results but they present two main deficiencies: (i) they are related to scenarios where the heterogeneity degree is very low; (ii) there are no systematic approaches to the real performance measurements.

In this paper we present the Service Condition (SC) concept as a mechanism to cope with the high level of network heterogeneity and useful when we need of a rigorous specification for the measuring, monitoring, and modeling of a particular real network condition. A preliminary and seminal definition of the $S C$ concept was provided in a network and service management framework in [9]. More precisely, we point our attention on the performance evaluation of some of the more interesting heterogeneous scenarios where there is the integration among LAN, GPRS, UMTS, and ADSL networks. The rest of the paper is organized as follow. Section 2 introduces and explains the $S C$ concept whereas Section 3 shows how this concept can be useful in a performance evaluation framework. Section 4 shows how we used the $S C$ concept to measure Quality of Service (QoS) parameters in real heterogeneous networks. Section 5 presents a summary of the experimental analysis results. Section 6 ends the paper with conclusion remarks.

\section{Network Heterogeneity and Service Condi- tion}

In pervasive and ubiquitous computing scenarios, several questions arise when we want to describe the way a service should be implemented to correctly fit requirements contained in a subscribed Service Level Agreement. A problem to cope with and, at the same time, an opportunity to ex- 
ploit is the "heterogeneity". Indeed, current networks represent a complex mixing of non-homogeneous variables:

- Heterogeneous network environments: Intermediate Systems, Access Networks, End Users Devices, Operating Systems (OSs), Service Type, ...

- Dynamic User Behavior: Handover, Handoff, MultiModal Applications, ...

For example, with respect to terminals, networks, and services we have: (i) Terminal heterogeneity, terminal devices can range from high-performance workstations, to Personal Digital Assistant (PDA), down to advanced mobile phones. (ii) Network heterogeneity, even if we consider as dynamically variable only the part that is closest to the user (i.e. the so called access or edge network), we have a quite large number of options to deal with. Wired (LANs, xDSL, ...), wireless (WLANs, Bluetooth, ...), and mobile networks (GPRS, EDGE, UMTS, ...) could be taken into account. (iii) Service heterogeneity, services may have different characteristics in terms of media involved (audio, video, ...), of their format (coding, compression, ...), and of their typology (synchronous, asynchronous, transactional, ...).

In this complex scenario, we introduce a rigorous specification to cope with: (i) Dynamic network changes; (ii) Dynamic user behavior; and (iii) Systematic measurement procedures. We called this rigorous concept "Service Condition". It is defined as a point in a multidimensional space composed of all considered key variables (access network, user device, operating system, application, time, ...). This point describes a particular combination of independent working conditions that characterize how a user accesses to a service. For example, in a 3-dimensional space composed of the Service Type, Access Network, and User Device sets, we have the result depicted in Figure 1.

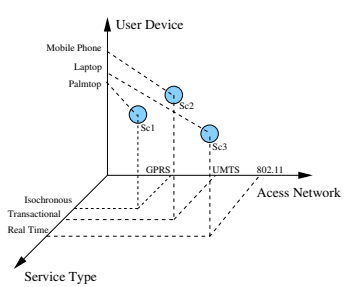

Figure 1. Service Conditions in a $3-D$ space

\subsection{Modeling End-to-End communications by us- ing the Service Condition concept}

In this Section we show how to use $S C$ concept to model real communications between end nodes. Let us consider a multidimensional space composed of four key variables:
1. access network $\in A N$ where:

$A N=\{$ GigaEthernet, Ethernet, WLAN 802.11b, GPRS, UMTS, ...

2. user device $\in E u D$ where: $E u D=\{$ Laptop, Palmtop, PC Desktop, Advanced Mobile Phone, Workstation, ... \}

3. operating system $\in O S$ where: $O S=\{$ Unix, Linux, Windows, Linux Familiar, ... $\}$

4. service type $\in S T$ where: $S T=\{$ Transactional, Real Time, Isochronous, ... $\}$

We have the following Service Condition Space

$$
\mathbf{S C}=(\mathbf{E u D}) \times(\mathbf{O S}) \times(\mathbf{S T}) \times(\mathbf{A N})
$$

A "Service Condition" represents a "4-tuple" in this 4dimensional space. Thus, in general, exploding this concept we have an $n$-dimensional space where ' $\mathrm{n}$ ' is the heterogeneity level or heterogeneity degree. Furthermore, if we consider as a variable also the time, we obtain an $(n+1)$ dimensional space. Therefore, from a practical point of view, by using the $S C$ concept, we are able to categorize and characterize network scenarios in which different combinations of end-users' devices, OSs and end-users' application (based on UDP and TCP) are taken into account, and in which different typologies of networks are present.

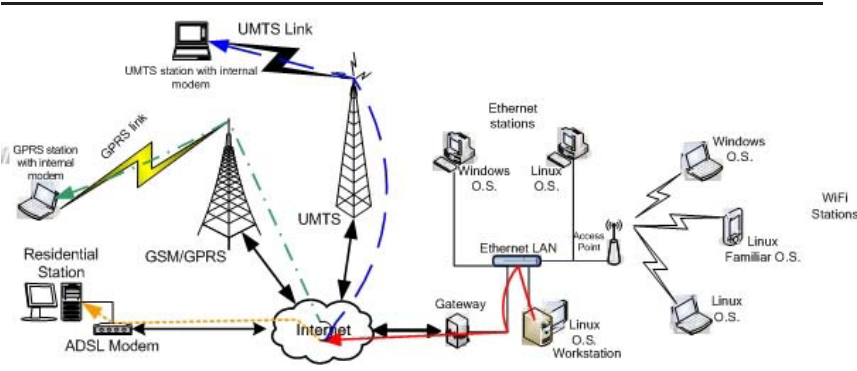

Figure 2. A real Heterogeneous Network

\section{Performance Evaluation and Service Con- dition}

The $S C$ concept may be very useful in performance evaluation studies and in particular, thanks to it, it is possible to draw a sort of cause vs. effect diagram. First, let us take into account a real scenario where two end systems need to communicate. For the sake of simplicity we can assume two ends among that present in Figure 2 where a real heterogeneous network is depicted. It represents the scenario where we performed our experimental analysis presented in Section 4 . The first step towards the definition of a systematic 
measurement approach is the definition of a network conceptual schema. All end to end communications can be collapsed in a same general schema. In Figure 3 such a conceptual schema is depicted. Two communication entities, a sender and a receiver, are directly connected through an IP network channel. Indeed, the end to end communications differ for the type of used network, its configuration, the type of host, and used OSs. By changing these parameters we define several strictly related $S C s$. The $S_{A}$ and $R_{A}$ legend are used in Figure 3 to indicate the sender and receiver application.

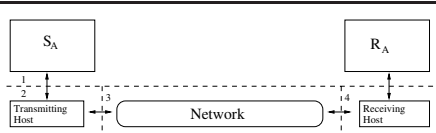

Figure 3. Network Conceptual Schema

Therefore, in a performance study of heterogeneous networks we can use $S C$ in the following way: we can model the communications between two ends by using the points of the SC space. More precisely, the single instances of the two ends will be represented by well defined points in the SC space (see Figure 1). Adopting this modus operandi we represent such communications by using relations between $S C$ points. For example, Figure 4 represents a situation where at one side there is a user that works in scenarios where several handoffs are possible and he is communicating (at receiver side) with a fixed server at own enterprise.

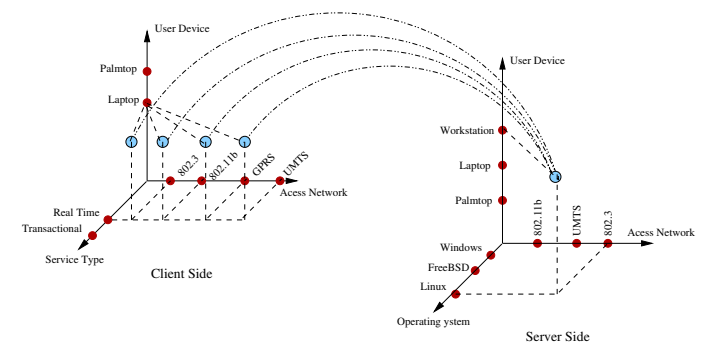

Figure 4. SCs in End to End communications

As for the defining of a sort of cause vs. effect diagram, by using tools for the network measurements [5] [6] we are able to completely characterize the end to end performance in terms of delay, packet loss, jitter, and throughput during the entire performance evaluation interval time. More precisely, by considering the SC Space as the independent multidimensional space and the QoS parameters space (in the following QoS space) as the dependent multidimensional space we define a close relationship between the current $S C$ and the measured QoS parameters. Due to the fact that the $S C$ at receiver side of Figure 4 is fixed, in Figure 5 the relation between the several Service Conditions and the perceived QoS parameters is depicted. By using this kind of association between SC space and QoS space we could also trace the sub-space of the $S C$ s that are linked to points in QoS space fulfilling a given application requirements ( $a d$ missibility sub-space). Also, thanks to the proposed concepts, we can simply derive the perceived QoS level associated to a particular $S C$. For istance, the SC concept and the related relationship with the QoS space could be useful to systematically study the complex network scenario presented in [10].

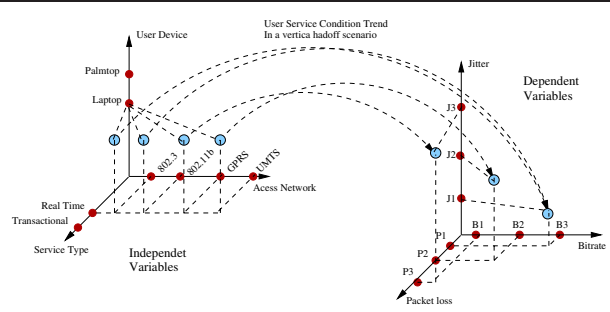

Figure 5. SCs and QoS parameters

In a heterogeneous scenario where handoffs are present, beside to a rigorous framework for performance evaluation, $S C$ could be used also to trace the user/network behavior and to determine the content adaptability.

\section{Experimental Analysis}

In this Section we show how to use the $S C$ concept to measure QoS parameters over heterogeneous IP networks. This analysis has been conducted by using D-ITG [5], a traffic generator capable of using a lot of random variables to profile the Inter Departure Time (IDT) and the Packet Size $(P S)$ of generated traffic packets. Moreover, D-ITG has the capability of logging both sent and received packets. Such a feature enables us to locate the testbed's part responsible for the measured performance, so it is possible to isolate the network from device dependencies. Before to step into experimental details, it is worth mentioning that we repeated each test several times. In the following graphics the mean values across 20 test repetitions are reported. We had a confidence interval greater than or equal to $95 \%$.

Due to space limitations, in this paper we focus just on three configurations among all the studied ones (Figure 2) [7]. We present the results first in a classical fashion, then by using the SC concept. The three configurations analyzed in following subsections are the ones highlighted in Figure 2 with dashed, dotted and dashed-dotted lines. The ana- 


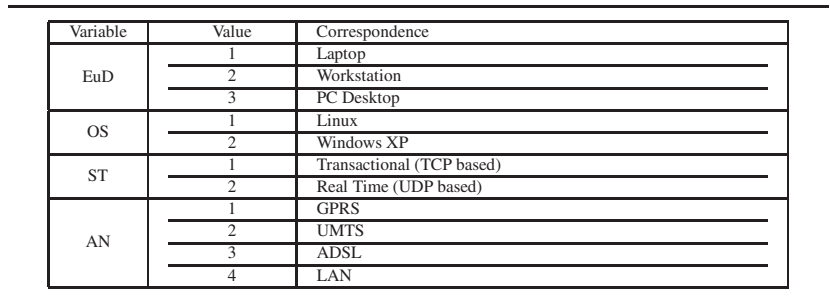

Table 1. Variables values and relative configuration for the considered SC space

lyzed configurations may be representative of real situations where users connected to the Internet are listening an Internet radio station which uses, in turn, HTTP for the audio streaming. For this situation we will consider the throughput and the jitter as QoS parameters because these ones are the most important in such a case [8]. This experimental analysis has been conducted by using probing traffic produced by using D-ITG and packets with $I D T=1 / 100 \mathrm{~s}$ and $P S \in\{64,128,256,512,1024\}$ Bytes.

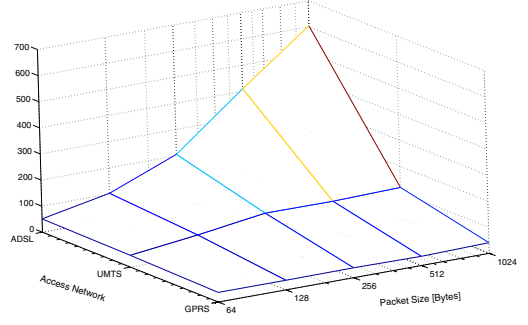

Figure 6. Bit rate vs packet size and AN

LAN $\rightarrow$ GPRS Scenario This first configuration is composed of a Windows XP equipped Laptop using TCP to communicate with a Linux workstation, where the first of them is a GPRS station and the other one is a LAN client. The configuration is the one depicted with a dashed-dotted (green) line in Figure 2. This configuration constitutes a point (said $s c_{1}^{2}$ ) in a $S C^{2}$ space (of dimension 8), where $S C^{2}$ is the Cartesian Product between two $S C$ spaces $\left(S C^{2}=S C \times S C\right)$. In particular, in mathematical terms:

$$
\begin{gathered}
s c_{s}^{1}=(2,1,1,4) ; s c_{r 1}^{1}=(1,2,1,1) \\
s c_{1}^{2}=\left(s c_{s}^{1}, s c_{r 1}^{1}\right)=(2,1,1,4,1,2,1,1)
\end{gathered}
$$

The correspondence between points in the SC space and relative configuration parameters can be found in Table 1.

LAN $\rightarrow$ UMTS Scenario This configuration is depicted with a dashed (blue) line in Figure 2. It also constitutes a point (said $s c_{2}^{2}$ ) in a $S C^{2}$ space. In mathematical terms:

$$
\begin{gathered}
s c_{s}^{1}=(2,1,1,4) ; s c_{r 2}^{1}=(1,2,1,2) \\
s c_{2}^{2}=\left(s c_{s}^{1}, s c_{r 2}^{1}\right)=(2,1,1,4,1,2,1,2)
\end{gathered}
$$

LAN $\rightarrow$ ADSL Scenario This last analyzed configuration is the one depicted with a dotted (orange) line in Figure 2. Its description in mathematical terms is.:

$$
\begin{gathered}
s c_{s}^{1}=(2,1,1,4) ; s c_{r 3}^{1}=(3,1,1,3) \\
s c_{3}^{2}=\left(s c_{s}^{1}, s c_{r 3}^{1}\right)=(2,1,1,4,3,1,1,3)
\end{gathered}
$$

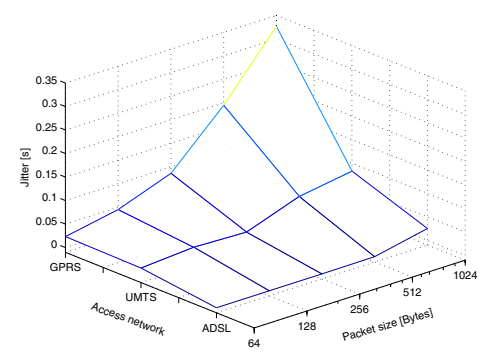

Figure 7. Jitter vs packet size and AN

\subsection{Experimental Results}

The following first explains experimental results in a classical fashion, then presents the same results using $S C$.

Figure 6 shows the bit rate measured in the three analyzed configurations with a three-dimensional plot. In it we can see that the maximum achieved bit rates were of 603.93 , 118.63, and 40.63Kbps with the ADSL, UMTS and GPRS respectively, all obtained with a $P S=1024$ Bytes. In Figure 7 the jitter measured in the three analyzed configurations is shown with a three-dimensional plot. We can see that the jitter attained values until $0.025,0.083$, and $0.327 s$ with the ADSL, UMTS, and GPRS respectively, all achieved with a $P S=1024$ Bytes .

In Figure 8 the best trade-off between bit rate and jitter are presented by using a $S C$ plot. In such a Figure the points of the independent variables space (SC space) are linked to the points of the dependent variables space (the QoS space). Due to the fact that the Service Condition at sender side is fixed, in Figure 8 the relation between the several SCs and the measured QoS parameters is depicted. With such a plot it is immediately visible the relationship between the configuration parameters and the measured QoS ones. Displaying the results in this way make it possible to easily recognize if a given $S C$ is suitable for a given application requirements. Indeed our sample application of the Internet radio has minimum bit rate and maximum jitter requirements, they change depending on required audio quality and on available buffers. The QoS points depicted in Figure 8 form a sub-space of the QoS space shown in Figure 9 composed of points that best fulfill considered application requirements. Therefore it can be used to identify the $a d$ missibility sub-space. In Figure 9 it is possible to see that 
in the GPRS case the point depicted in Figure 8 was obtained with a $P S=64$ Bytes. In the UMTS case the best trade-of point was obtained with a $P S=256$ Bytes. Finally in the ADSL case it was achieved with the maximum used $P S=1024$ Bytes.

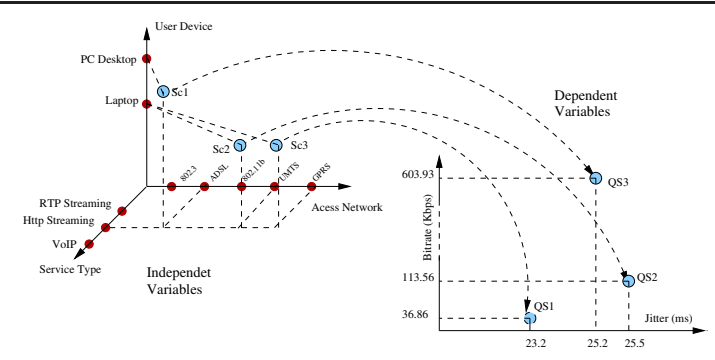

Figure 8. "Service condition" plot

\section{Summary of Results}

As previously said we tested a large number of heterogeneous network configurations. In this paper we point our attention to the three depicted in Section 4. For a complete results analysis refer to [7]. In this section, we report a summary of results of the complete analysis of scenarios where also PDA and WLANs are present. Thanks to the use of our systematic measurement approach we are in charge of listing some lessons we learned: (i) PDA (at both sender and receiver sides) presents very low performance with several NICs; (ii) in TCP case, at sender side the maximum throughput with $802.11 \mathrm{~b}$ is equal to $1.1 \mathrm{Mbps}$; (iii) GPRS presents very high RTT and jitter values. These parameters reach their maximum value in the case of GPRS $\rightarrow$ UMTS (and vice versa); (iv) in the case of $802.11 \mathrm{~b}$ with two stations, the ad-hoc mode configuration presents performance greater than the infrastructural configuration (with AP); (v) up to $P S<512 b y t e s$, TCP reaches better performance than UDP; (vi) GPRS and UMTS networks present the best performance with PS greater than 512 bytes. In the case of PS equal to 32 bytes and 64 bytes, the GPRS connection is intermittent (we experimented several network flappings).

\section{Conclusion}

In this paper we presented some of our measurements of QoS parameters over real networks characterized by a high level of heterogeneity in terms of access networks, end user devices, operating systems, and protocols. Furthermore, thanks to the proposed Service Condition concept we have depicted the relation between different $S C s$ and the relative QoS parameters. Therefore, we have shown the importance of setting up a precise framework for the performance

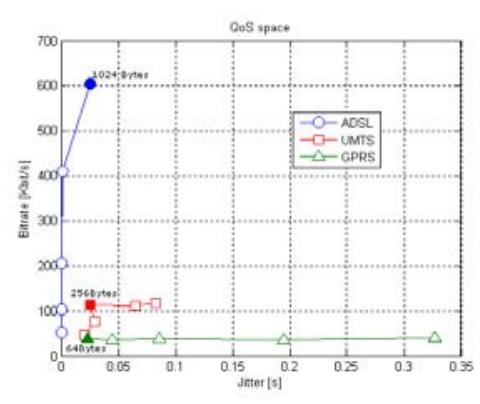

Figure 9. QoS space plot for all PS

evaluation of a complex scenario like that of Figure 2 where a large number of $S C s$ are involved. Along with the proposal of a novel concept to model network conditions (that can be used also in other field of networking such as network management, network traffic modeling, network security, ...) we found the best PS as function of the analyzed SCs. Finally, the presented results can be also used as performance references for development of wireless communication applications over multiservice and heterogeneous networks.

\section{References}

[1] J. P. Singh, N. Bambos, B. Srinivasan, D. Clawin,, "Wireless LAN performance under varied stress conditions in vehicular traffic scenarios", Proc. of VTC, 2002.

[2] A. Messier, J. Robinson, K. Pahlavan, "Performance monitoring of a wireless campus area network", Proc. of Local Computer Networks, 1997.

[3] G. Xylomenos, G. C. Polyzos, "TCP and UDP performance over a wireless LAN", Proc. of INFOCOM 99.

[4] J. C. Amaro, R. P. Lopes, "Performance analysis of a wireless MAN", Network Computing and Applications, 2001.

[5] http://www.grid.unina.it/software/ITG

[6] http://www.slac.stanford.edu/xorg/nmtf/ nmtf-tools.html

[7] A. Botta, D. Emma, S. Guadagno, A. Pescapé, "Performance Evaluation of Heterogeneous Network Scenarios", Dipartimento di Informatica e Sistemistica Technical Report TR-DIS-102004, October 2004 http://www.grid.unina.it/software/ITG/ D-ITGpubblications/TR-DIS-102004.pdf

[8] M. Alves, L. Corsello, D. Karrenberg, C. Ogut, et al., "New Measurements with the RIPE NCC Test Traffic Measurements Setup,", Proc. of PAM 2002, pp. 66-75.

[9] M. D’Arienzo A. Pescapé, and G. Ventre, "Dynamic Service Management in Heterogeneous Networks", International Journal of Network and System Management (JNSM), Vol. 12, No. 3, Sep. 2004, ISSN 1064-7570

[10] H.J. Lee, M.S. Kim, J.W. Hong and G.H. Lee "QoS Parameters to Network Performance Metrics Mapping for SLA Monitoring”, KNOM Review, Vol. 5, No. 2, December 2002. 